\title{
DESENVOLVIMENTO MÓVEL HÍBRIDO
}

\author{
HYBRID MOBILE DEVELOPMENT
}

\author{
Kelly Cristina Venteu - kellyventeu@gmail.com \\ Giuliano Scombatti Pinto - giuliano.pinto@fatectq.edu.br \\ Faculdade de Tecnologia de Taquaritinga (FATEC) - SP - Brasil
}

DOI: 10.31510/infa.v15i1.337

\section{RESUMO}

O desenvolvimento de aplicativos móveis intensificou-se nos últimos anos devido ao crescimento acelerado e popularização dos smartphones. Atualmente existem dois sistemas operacionais móveis e cada um possui sua loja de aplicativos e ambientes de desenvolvimento. No entanto, quanto mais sistemas diferentes existem, maior o esforço, custo e tempo para desenvolver um app para todas as plataformas existentes. Surgiu então, o conceito de desenvolvimento multiplataforma ou híbrido, com a ideia de codificar apenas uma vez e envolver várias plataformas. Com o objetivo de conhecer o que é o desenvolvimento mobile híbrido e entender mais sobre quais as diferenças entre o desenvolvimento híbrido e o nativo, explorar as tecnologias utilizadas para o seu desenvolvimento (HTML, CSS e Java Script) e inteirar-se dos frameworks utilizados (Ionic, PhoneGap e Cordova). A metodologia básica utilizada para este estudo será bibliográfica, com referencial teórico baseado em livros e websites. Contudo, este trabalho obteve um resultado definindo a vantagem do desenvolvimento híbrido quando comparada com o desenvolvimento nativo, bem como apresentar as principais dificuldades enfrentadas pelos desenvolvedores em relação à diversidade nos tipos de desenvolvimento e as plataformas existentes.

Palavras-chave: Desenvolvimento App. Híbrido. Mobile. Desenvolvimento móvel. Web.

\begin{abstract}
The movement of mobile applications has intensified in recent years due to the accelerated growth and popularization of smartphones. Injectors there are two operating spaces and each has its application store and development environments. No longer, what more the patterns different there, major the effort, cost and progress for development to all the existent platform. Then came the concept of multiplatform or hybrid development, with an idea of coding only once and several platforms. In order to understand the development of the hybrid hybrid and to understand it more as the differences between hybrid and native development, to explore the technologies used for its development (HTML, CSS and Java Script) and to know the frameworks used Ionic, PhoneGap and Cordova). The basic methodology used for this study will be bibliographical, with theoretical reference based on books and websites. However, this work has achieved a result by defining the advantage of hybrid development as compared to native development as well as presenting the main difficulties faced by developers regarding the diversity in the types of development and the existing platforms.
\end{abstract}

Keywords: App Development. Hybrid. Mobile. Mobile Development. Web. 


\section{INTRODUÇÃO}

Atualmente, os telefones, smartphones, dispositivos móveis e a utilização de aplicativos conquistam cada vez mais tempo e espaço na vida das pessoas. Conforme Fontes (2016), em uma pesquisa realizada pela Fundação Getúlio Vargas, existem 244 milhões de dispositivos

móveis conectados à Internet no Brasil. É quase impossível viver atualmente com a variedade modelos de aparelhos de celulares, sem a utilização de aplicativos com suas inúmeras funções de comunicação, já que hoje o Brasil é o país com o mercado de aplicativos móveis mais competitivo do mundo.

Esses dispositivos oferecem conectividade e capacidade de uso em qualquer lugar e em qualquer momento, sendo essenciais, tanto para uso pessoal, quanto profissional. Para o desenvolvimento de aplicações para tais dispositivos deve-se ter a noção do objetivo e qual sistema operacional ele atuará, pois cada aplicativo é desenvolvido em sua linguagem exclusiva, com suas devidas plataformas e sistemas operacionais.

De acordo com Muller (2016), não é vantajoso para o desenvolvedor criar diversos códigos fontes para atender cada plataforma móvel, pois isto pode consumir mais tempo. Diante deste fator, o presente trabalho, procura mostrar uma forma eficaz de resolver essa questão, utilizando apenas uma plataforma de desenvolvimento que abrange todos os sistemas operacionais, mais conhecido como desenvolvimento híbrido. Este trabalho deve apresentar as vantagens e a comparação entre os tipos de desenvolvimentos de aplicações para dispositivos móveis, de forma a destacar o motivo pelo qual atualmente aplicativos híbridos têm sido grandemente utilizados e quais são os principais aspectos que tornam um aplicativo nativo inviável para certos ambientes.

\section{AMBIENTE DE DESENVOLVIMENTO MÓVEL}

Os aplicativos móveis (apps) são produtos projetados e desenvolvidos para serem executados especificamente em dispositivos eletrônicos móveis, como tablets, notebooks, celulares e smartphones, com larga capacidade de armazenamento e processamento. Um aplicativo móvel pode ser baixado diretamente do aparelho eletrônico, desde que o dispositivo possua conexão com a Internet. A gama de fornecedores que disponibilizam aplicativos para 
download através de lojas virtuais como a Apple Store - loja virtual da Apple, Play Store - loja virtual do Google para o sistema operacional Android.

\subsection{Aplicativos.}

Conforme Morimoto (2009), os smartphones possibilitam a concentração de um grande volume de funcionalidades. Existem diversos tipos de aplicativos alguns mais utilizados e com melhor facilidade, do que outros. Cada aplicativo é um software com funções específicas em dispositivos móveis. O mercado de dispositivos móveis é ampliado por diversos fabricantes, incluindo uma série de plataformas de desenvolvimento, sistemas operacionais, software e hardware. A existência de múltiplas plataformas possibilita uma grande variedade de aplicativos, cada um codificado para executar em sua arquitetura específica.

Conforme Coelho (2015), surge então a tecnologia híbrida, que é capaz de transformar o que seria desenvolvimento de um site ou uma aplicação Web, em uma aplicação móvel, com a capacidade de ser executada em qualquer dispositivo móvel.

\subsection{Diferenças entre aplicação nativa e híbrida}

Ao pensar em criar um aplicativo, deve-se pensar que tipo de aplicativo desenvolver, nativo ou híbrido. $\mathrm{O}$ aplicativo deve oferecer uma boa experiência com o usuário, sendo necessário analisar qual o tipo de desenvolvimento vai suprir tais necessidades. No entanto, as duas opções apresentam tempos diferentes de desenvolvimento de acordo com o objetivo e custo.

De acordo com Madureira (2017), o aplicativo nativo é programado na linguagem de cada sistema operacional, como Java no Android e Objective-C no iOS, cada plataforma apresentando suas próprias ferramentas e elementos de interface. Mediante essas características, o app nativo é desenvolvido para utilização em uma plataforma específica, como iOS ou Android, sendo capaz de explorar todas as potencialidades da plataforma para a qual foi criado, conseguindo ter acesso a diversos recursos dos aparelhos como GPS, câmera, calendário, lista de contatos, entre outros. É importante salientar que nem sempre os aplicativos nativos precisam da internet para seu funcionamento. Madureira (2017) afirma ainda que os desenvolvedores respeitam a um padrão de design, que são oferecidos para cada sistema operacional, como um guia. 
Não é aprovada a ideia de ter que repetir todo o trabalho mais de uma vez em linguagens de programação diferentes. Além da perda de tempo em fazer o trabalho, falhas encontradas posteriormente provavelmente também terão que ser corrigidas mais de uma vez (MADUREIRA, 2017, n.p.).

O desenvolvimento do aplicativo nativo leva tempo e é mais trabalhoso, pois será necessário desenvolver um app para cada plataforma distinta. Além disso, os códigos não podem ser reutilizados, pois cada plataforma requer diferentes códigos para o desenvolvimento dos aplicativos e mão de obra diferenciada. Ramires (2017) afirma que o app nativo tem um custo mais elevado, devido a sua linguagem própria e específica de cada plataforma. Entretanto, se o público-alvo do aplicativo é mais exigente, buscando rapidez e confiabilidade, o app nativo é, a melhor opção para atender essa demanda.

Contudo o desenvolvimento de um app híbrido acaba sendo mais rápido e também mais barato porque o código desenvolvido apenas uma vez e pode ser distribuído em várias plataformas. A redução de desenvolvimento no quesito tempo não tem comparação aos aplicativos nativos e se deve à possibilidade de execução do aplicativo híbrido em diferentes plataformas, não existindo a necessidade de desenvolver o aplicativo várias vezes para se adequar a distintas plataformas. O desenvolvimento híbrido é uma ótima opção para situações onde não há necessidade de alta performance do aplicativo, pois não funcionam tão rápido quanto um aplicativo nativo (MADUREIRA, 2017, n.p.).

O aplicativo híbrido pode ser construído utilizando as linguagens HTML5, CSS e JavaScript, assim como um site mobile. Esse código é alocado dentro de um container, integrando as funcionalidades que o seu dispositivo oferece (MADUREIRA, 2017, n.p.). Uma vez que se utiliza um framework específico, o código desenvolvido com tecnologia web pode ser compilado para diferentes plataformas. A manutenção também se torna mais barata uma vez que a mão de obra é mais genérica e fácil de ser encontrada no mercado. 
Ilustração 1 - Diferenças entre o desenvolvimento híbrido e nativo

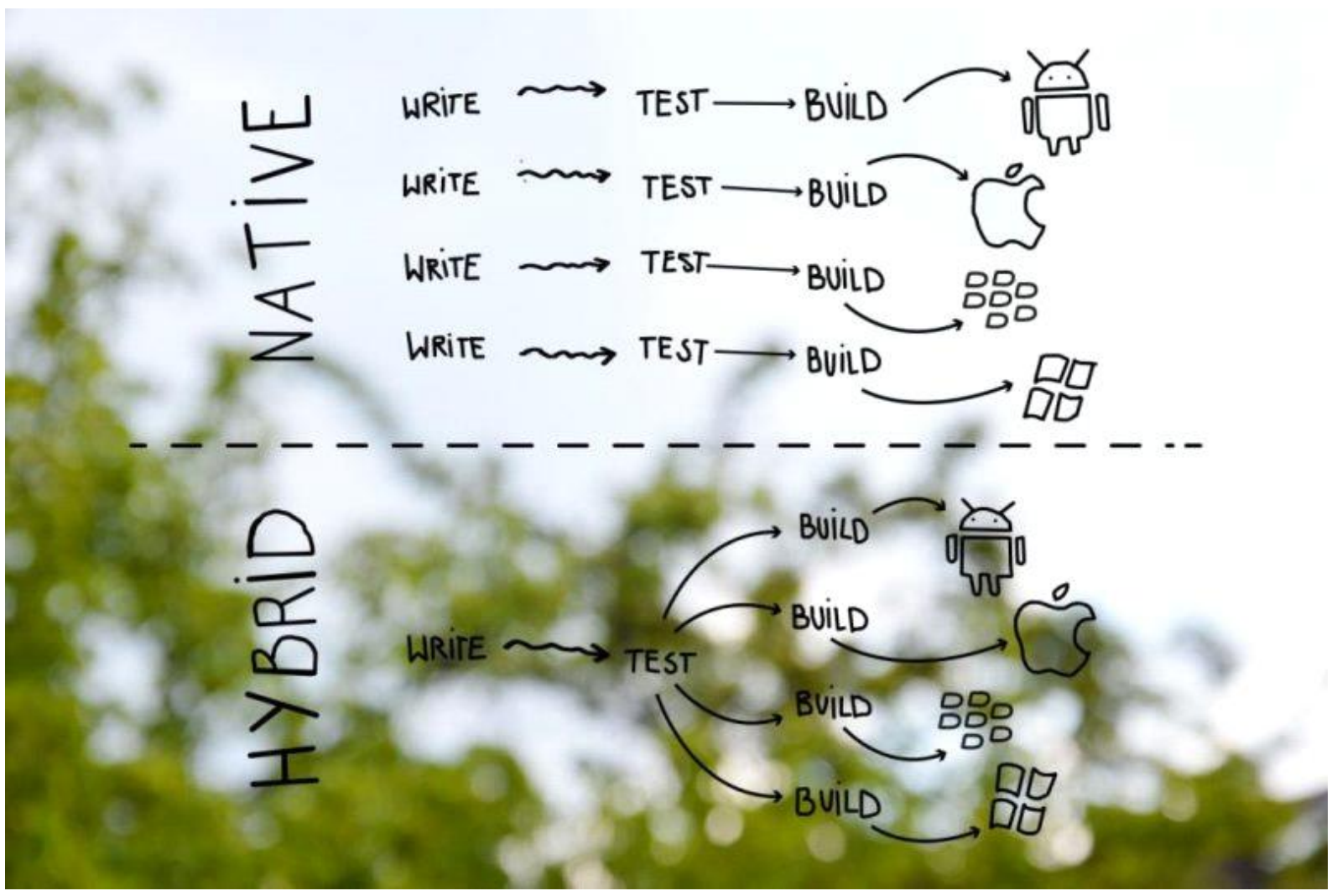

Fonte: RADEJ (2017)

Analisando a ilustração 1, observa-se que uma das características que mais diferenciam o aplicativo nativo do aplicativo híbrido é o processo de desenvolvimento. Para desenvolver uma aplicação nativa, como mencionado na definição e bem onisciente na ilustração, cada dispositivo tem seu próprio processo de desenvolvimento exclusivo. Em contrapartida para desenvolver uma aplicação híbrida, o desenvolvimento é iniciado com uma única programação, essa programação é testada e compilada de forma única, e atribuí simultaneamente às plataformas, caso o desenvolvedor queira acrescentar e alterar algum detalhe ou requisito do aplicativo, apenas modifica-se todos os códigos fonte, de uma só vez, não precisando alterar todos, um a um.

\section{TECNOLOGIAS UTILIZADAS NO DESENVOLVIMENTO HÍBRIDO.}

As tecnologias mais utilizadas para esse desenvolvimento híbrido são HTML, CSS e JavaScript (MADUREIRA, 2017, n.p.). Partindo desse conceito de junção dessas principais tecnologias, a utilização das linguagens de programação da web, resultará no funcionamento de 
um aplicativo em diferentes plataformas. Assim, a seguir serão descritas algumas características dessas tecnologias.

\title{
3.1 Hyper Text Markup Language
}

O Hyper Text Markup Language (HTML), significa Linguagem de Marcação de Hipertexto, uma linguagem básica para os websites. Silva (2014, p. 22-24) afirma que pode-se resumir hipertex to como todo o conteúdo inserido em um documento para web e que tem como característica a possibilidade de interligar a outros documentos da web. Documentos marcados são constituídos de títulos, parágrafos, listas, tabelas e figuras. Assim, o HTML possui códigos para criar páginas na web.

\begin{abstract}
Ao criar um documento em um processador de textos, pode adicionar estilos ao texto para explicar a estrutura do documento. Por exemplo, pode distinguir cabeçalho do corpo principal do texto usando um cabeçalho. Pode inserir também tabelas no documento para armazenar dados, ou criar listas com marcadores para uma série de tópicos relacionados e assim por diante. Embora isto não afete a apresentação do documento, o propósito principal deste tipo de marcador é fornecer uma estrutura que torna o documento mais fácil de entender. (DUCKETT, 2010, p. 3)
\end{abstract}

Há diversos conjuntos de caracteres ' $<$ ' e ' $>$ ' envolvendo palavras ou letras entre eles, como $\langle$ html $>$, <head $>$, </title > e </body> (DUCKETT, 2010, p. 4). Estes sinais e palavras dentro deles são conhecidos como rótulos e são marcadores. Cada etiqueta tem seu significado próprio. Portanto, o HTML não é mais do que uma série de tags que se utiliza para definir a forma ou o estilo que se deseja aplicar no documento.

\subsection{Cascading StyleSheets}

O Cascading Style Sheets (CSS), que significa folhas de estilo em cascata, permitem controlar a apresentação de conexões, cores de fundo, estilos de listas e contornos de caixas (DUCKETT, 2010, p. 327). Como é uma linguagem de folhas de estilos é utilizada para formatar os documentos escritos na linguagem de marcação.

É usada para controlar como deve ser a aparência de um documento. Por exemplo, pode usá-la para especificar que um tipo de fonte deve ser grande, negrito, arial ou que o fundo de uma página deve ser verde claro (DUCKETT, 2010, p. 25). Enquanto o HTML é usado para 
estruturar os conteúdos, o CSS é utilizado para dar estilo e variações deixando-os mais elegante e atrativos para o usuário.

\subsection{JavaScript}

JavaScript é uma linguagem de programação utilizada em páginas web e aplicativos. Um dos seus objetivos é o controle do HTML e o CSS manipulando os comportamentos das páginas.

O JavaScript serve para adicionar interatividade às páginas web que cria e para funcionar com o navegador exibindo a página web (DUCKETT, 2010, p. 25).

São infinitas funcionalidades, como manipulando conteúdo e apresentação, interagindo o navegador com formulários, fornecendo aos desenvolvedores uma linguagem de programação, que é utilizada em páginas web e permite a execução de algumas tarefas.

Pode-se escrever marcação HTML e inseri-la na marcação de um documento existente. Por exemplo: inserção de conteúdos diferenciados e escolhidos de acordo com o navegador do usuário. [...] É capaz de definir, alterar e controlar de forma dinâmica a apresentação de um documento HTML, como aspectos relacionados a cor de fundo. É possível manipular a folha de estilos associada ao documento criando novas regras CSS ou anulando regras existentes. [...] Podemos controlar o comportamento do navegador em diversos aspectos, como criar janelas pop-up, apresentar mensagens ao usuário, alterar as dimensões do navegador, interferir na barra de status, retirar menus, fechar e abrir janelas. [...] Java Script é capaz de acessar os campos e valores digitados em um formulário HTML e proceder a validação dos dados, realizar cálculos e fornecer dicas de preenchimento dos campos (SILVA, 2010, p 24-25).

O JavaScript é a linguagem que vai fazer essa informação receber alguns comportamentos, propriamente, onde cálculos são executados sobre dados e decisões são tomadas programaticamente baseadas em alguma entrada que o programa receber (DUCKETT, 2010, p 483).

\section{FRAMEWORKS UTILIZADOS PARA O DESENVOLVIMENTO HÍBRIDO}

Framework é uma "base" de onde se pode desenvolver algo maior ou mais específico. Uma coleção de códigos-fonte, classes, funções, técnicas e metodologias que facilitam o desenvolvimento de novos softwares(MINETTO, 2007, p. 17). Ou seja, é conjunto de códigos 
prontos, que possui um conjunto de funções implementadas em uma linguagem de programação e usada para auxiliar e facilitar o desenvolvimento de software,

Framework é o ambiente usado para criação de softwares como um serviço. A fim de poupar tempo e trabalho e aumentar a produtividade do desenvolvedor ao desenvolver uma aplicação existem os frameworks, que possuem diversas funcionalidade, testadas e implementadas por outros desenvolvedores (MICROSOFT, 2001, n.p.).

Nas próximas subseções serão descritos alguns frameworks utilizados para o desenvolvimento multiplataforma.

\subsection{Ionic}

Ionic é um framework criado no final de 2013 para a construção de aplicativos híbridos em dispositivos móveis. Ele vem com um conjunto de componentes e funções que podem ser utilizadas para criar aplicações mobile totalmente funcionais e atrativas.

O Ionic é um framework free e open source totalmente otimizado para criar aplicativos híbridos com html5, css e javascript para iOS e Android, foi criado com base no AngularJs e possui vários componentes e ferramentas que facilitam o desenvolvimento e não prejudicam a performance do seu aplicativo (FRANCO, 2017, n.p.).

Franco (2017) afirma que precisa-se "informar para qual plataforma o aplicativo irá rodar, depois executar o aplicativo em um emulador, em um dispositivo conectado ao computador ou em um navegador. Por último gerar a versão executável do aplicativo.”. Esses são os passos que deve-se seguir para produzir o aplicativo.

A linguagem utilizada para o desenvolvimento no ionic é o JavaScript, e usa-se HTML e CSS para criação da interface gráfica. Utiliza-se qualquer IDE (Integrated Development Environment), é o ambiente de desenvolvimento integrado, que integra as várias ferramentas necessárias para o desenvolvimento de softwares, de acordo com as preferências do programador. O Ionic é um framework muito robusto e possui uma curva de aprendizado muito baixa. (FRANCO, 2017, n.p.). Com ele, é possível construir aplicativos poderosos e com um ótimo desempenho, existindo uma ampla quantidade de recursos para facilitar o desenvolvimento. 


\subsection{PhoneGap e Cordova}

O PhoneGap e o Cordova são frameworks de desenvolvimento que permitem criar aplicativos híbridos usando apenas as tecnologias JavaScript, HTML 5 e CSS, para diferentes plataformas, sendo gratuitos e open sources, foram criados pela empresa Nitobi.

A Adobe comprou a Nitobi e doou o código fonte para a Apache que nomeou o projeto para Apache Cordova. A Adobe usa essa base para prover um Cordova incrementado (MACHADO, 2016, n.p.). Compreende-se que ambos são os mesmos frameworks entre plataformas com nomes diferentes. O Phonegap é sustentado pelo apache Cordova. Phonegap é Cordova, com algumas habilidades extra da Adobe. Se estiver desenvolvendo um aplicativo móvel híbrido, pode-se criá-lo usando Cordova ou escolher o ecossistema da Adobe para usar a distribuição PhoneGap de Cordova.

As primeiras versões do PhoneGap exigiam que, para se criar aplicativos para a plataforma desejada, deveria produzi-la na mesma plataforma. A Adobe lançou então, o PhoneGap Build, um serviço integrado com outras ferramentas. O Phonegap Build, além do ambiente ser na nuvem também compila o seu código para diferentes plataformas, mas ambos não trabalham com elementos visuais (MACHADO, 2016, n.p.). Trata-se de uma plataforma web para empacotamento e criação de aplicativos utilizando a tecnologia PhoneGap.

A fim de se evitar confusões em relação aos nomes dados ao PhoneGap e Cordova, entende-se o PhoneGap como uma distribuição do Apache Cordova, mantido pela Adobe.

Proporcionando a vantagem da tecnologia criada por uma equipe de desenvolvedores robusta - além do acesso ao conjunto de ferramentas PhoneGap, para que você possa chegar a móvel mais rápido. O PhoneGap usa tecnologias web baseadas em padrões para conectar aplicativos da Web e dispositivos móveis. Uma vez que os aplicativos PhoneGap são compatíveis com os padrões, eles são à prova de futuro para trabalhar com navegadores à medida que evoluem. Baixado milhões de vezes e está sendo usado por centenas de milhares de desenvolvedores. Milhares de aplicativos criados usando o PhoneGap estão disponíveis em lojas e diretórios de aplicativos para dispositivos móveis (PHONEGAP, 2018, n.p.).

A PhoneGap possui bibliotecas que possibilitam uma maior facilidade para manipular componentes nativos, permitindo o desenvolvendo de um único código para várias plataformas.A aplicação híbrida produzida é recomendada apenas para projetos de pequeno e médio porte, devido a sua performance ser reduzida. 


\section{CONCLUSÃO}

O presente trabalho apresentou uma abordagem sobre as possíveis formas de desenvolvimento de dispositivos móveis, com foco no desenvolvimento híbrido. Sucedeu-se uma reflexão sobre as dificuldades enfrentadas pelos desenvolvedores em relação à diversidade nos tipos de desenvolvimento e as plataformas existentes.

Com aplicativos híbridos, é possível codificar apenas uma vez e distribuir o código gerado para diversas plataformas reduzindo assim o tempo e o custo diferentemente do desenvolvimento de aplicativos nativos. Com o adicional do alcance no número de plataformas se torna mais atrativa.

No que se refere à performance, a utilização de aplicações híbridas tende a ser mais lenta que as nativas, pelo fato de utilizar as linguagens HTML, CSS e JavaScript ao invés de utilizarem a linguagem nativa para o dispositivo. Entretanto, torna o tempo de desenvolvimento mais curto, além de tornar a aplicação multi-plataforma com pouco trabalho de adaptação.

Contudo, as aplicações híbridas possibilitam uma redução de tempo quando comparadas ao desenvolvimento de aplicações nativas, pois, possibilitam que o código seja escrito apenas uma vez e possa ser utilizado em outras plataformas, o que não ocorre no desenvolvimento nativo.

\section{REFERÊNCIAS}

COELHO, Pedro. Desenvolvimento Móvel com HML5. Lisboa: FCA, 2015.

DUCKETT, Jon. Introdução à programação Web com HTML, XHTML e CSS. Rio de Janeiro: Ciência Moderna Ltda, 2010.

FONTES, Henrique. Mercado de aplicativos cresce no Brasil e alunos da USP em São Carlos conquistam espaço no cenário. Jornal da USP. São Carlos, 21 set. 2016. Disponível em: $<$ http://jornal.usp.br/universidade/mercado-de-aplicativos-cresce-no-brasil-e-alunos-da-uspem-sao-carlos-conquistam-espaco-no-cenario/>. Acesso em: 01 out, 2017.

FRANCO, Felipe.Como criar aplicativos com Ionic Framework. Fábrica de Código. 20 jun. 2017. Disponível em: <http://www.fabricadecodigo.com/como-criar-aplicativos-com-ionicframework/> Acessado em: 06 out, 2017

MACHADO, Diogo Souza. Apache Cordova e Phonegap e suas pequenas diferenças. Diogo Souza Machado. 14 jun.2016. Disponível em: <https://www.diogomachado.com/2016/06/14/apache-cordova-e-phonegap-diferencas.html> Acessado em: 07 fev, 2018. 
MADUREIRA, Daniel. Aplicativo nativo, web App ou aplicativo híbrido? .Net.8 mar. 2017. Disponível em: <https://usemobile.com.br/aplicativo-nativo-web-hibrido/>. Acesso em: 10 out, 2017.

MICROSOFT. O sistema do .NET Framework. .Net. 10 jun. 2001. Disponível em: <https://docs.microsoft.com/pt-br/dotnet/framework/get-started/system-requirements> Acessado em: 05 out, 2017.

MINETTO, Elton Luis. Frameworks para Desenvolvimento em PHP. São Paulo: Novatec Editora Ltda, 2007.

MORIMOTO, Carlos Eduardo.Smartphones Guia Prático. Porto Alegre: Sul Editores, 2009.

MULLER, Leonardo. Plataforma da Intel permite desenvolver para Android e iOS ao mesmo tempo TecMundo. 28 jul. 2016. Disponível em: $<$ https://www.tecmundo.com.br/programacao/107831-plataforma-intel-permite-desenvolverandroid-ios-mesmo-tempo.htm> Acessado em: 02 out, 2017.

PHONEGAP. Um resumo de alto nível sobre o que é o PhoneGap. Adobe PhoneGap. 27 jan. 2018. Disponível em <https://phonegap.com/> Acessado em: 07 fev, 2018

RADEJ, Pawel. 4 pontos principais porque as aplicações híbridas são lucrativas. eCodile. 21 fev. 2017.Disponível em: <http://ecodile.com/2017/02/21/4-major-points-why-hybridapplications-are-profitable/ > Acesso em: 01 out, 2017

RAMIRES, Thammy. Qual a diferença entre web app, app nativo e app híbrido? FabApp. 25 jan. 2017 .Disponível em: <http://fabricadeaplicativos.com.br/aprender/qual-diferenca-entreweb-app-app-nativo-e-aplicativo-hibrido/> Acessado em: 05 out, 2017

SILVA, Maurício Samy. HTML5 A linguagem que revolucionou a web. São Paulo: Novatec Editora Ltda, 2010.

SILVA, Maurício Samy. JavaScript guia do programador. São Paulo: Novatec Editora Ltda, 2014. 